\title{
Methods in medicine
}

\section{Self ligating lingual appliance}

\author{
Maj Pankaj Juneja ${ }^{a}$, Col S.S. Chopra, ${ }^{b, *}$, Col B.K. Jayan ${ }^{c}$ \\ ${ }^{a}$ Graded Specialist (Orthodontics), MDC Secundrabad, (AP), India \\ ${ }^{\mathrm{b}}$ Senior Specialist (Orthodontics), Department of Dental Surgery, AFMC, Pune 40, India \\ ${ }^{c}$ Associate Professor(Orthodontics), Department of Dental Surgery, AFMC, Pune 40, India
}

\section{A R T I C L E I N F O}

Article history:

Received 6 March 2012

Accepted 13 July 2012

Available online 23 October 2012

Keywords:

Lingual orthodontics

Self ligating

Indirect bonding

\begin{abstract}
A B S T R A C T
Adult demand for orthodontics has grown considerably over the past 10 years propelling increased demand for Esthetic Orthodontics. Lingual appliances are a viable option toward providing Esthetic Orthodontics. The lingual surface of the teeth has a unique morphology that makes it difficult to place brackets in ideal positions. Indirect bonding has become the established methods of overcoming these discrepancies, along with the latest designs of self ligating brackets which offer more efficient mechanics and shorter treatment time.
\end{abstract}

(c) 2012, Armed Forces Medical Services (AFMS). All rights reserved.

\section{Introduction}

Esthetic concerns continue to remain responsible for a significant segment of patients seeking orthodontic treatment. Research has shown that physically attractive people achieve higher levels of success in many aspects of life than unattractive people. ${ }^{1}$

Requests based on esthetic needs are on the increase in many clinical fields, particularly in dentistry. Orthodontics is no exception. Many adult patients, requiring orthodontics for esthetic and functional (periodontal, preprosthetical, temporomandibular joint) reasons, are discouraged by visible orthodontic treatment. Lingual orthodontics is the esthetic solution to this problem. ${ }^{2}$

\section{Case selection}

All cases indicated for conventional labial orthodontic therapy may be taken up for lingual appliance therapy. However, the anchorage planning for lingual orthodontics is different from labial orthodontics because large amount of anchorage is available in lingual orthodontics, especially in the lower arch. Vectors of orthodontic forces applied to lingual brackets pass lingual to the centers of rotation of the teeth, which increases lingual crown torque to the anterior teeth and forces posterior teeth in an upright position. Clinical examination, study models, lateral cephalograms, orthopantomograms and intra- \& extra-oral photographs are the essential pre-treatment records (Fig. 1).

\section{Set up}

Set up models are essential for lingual orthodontic treatment; they provide a means of identifying plans and goals of treatment and determine bracket position. The method of lingual bracket placement takes into account the anatomical discrepancies in the lingual surfaces of the teeth. This is accomplished by first constructing an ideal diagnostic set up from a duplicate set up model of the patient original

\footnotetext{
* Corresponding author. Tel.: +91 9637857944.

E-mail address: drsschopra@hotmail.com (S.S. Chopra). 

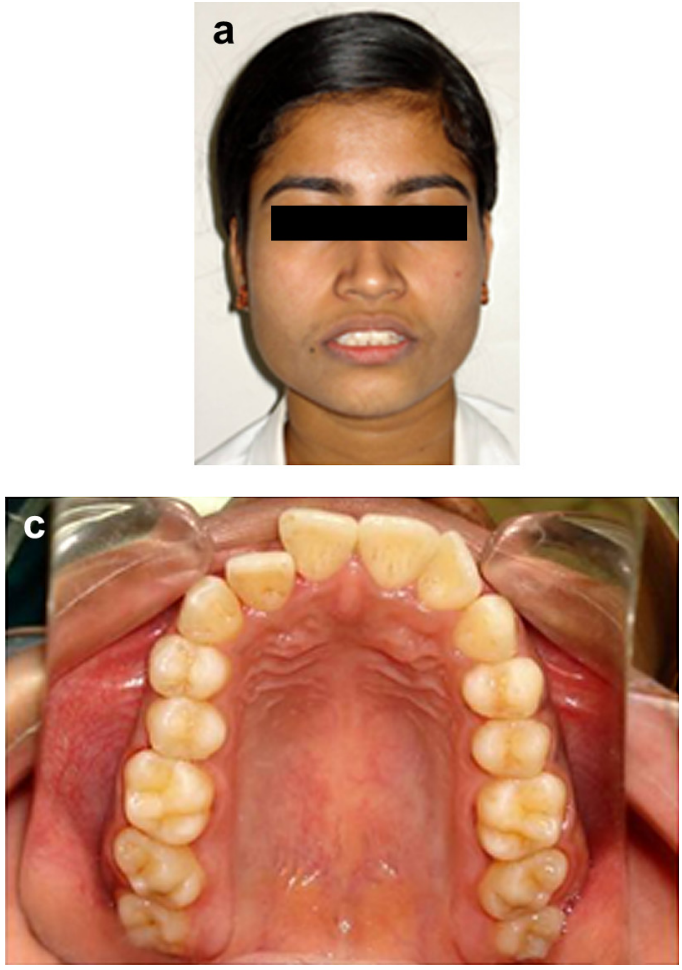
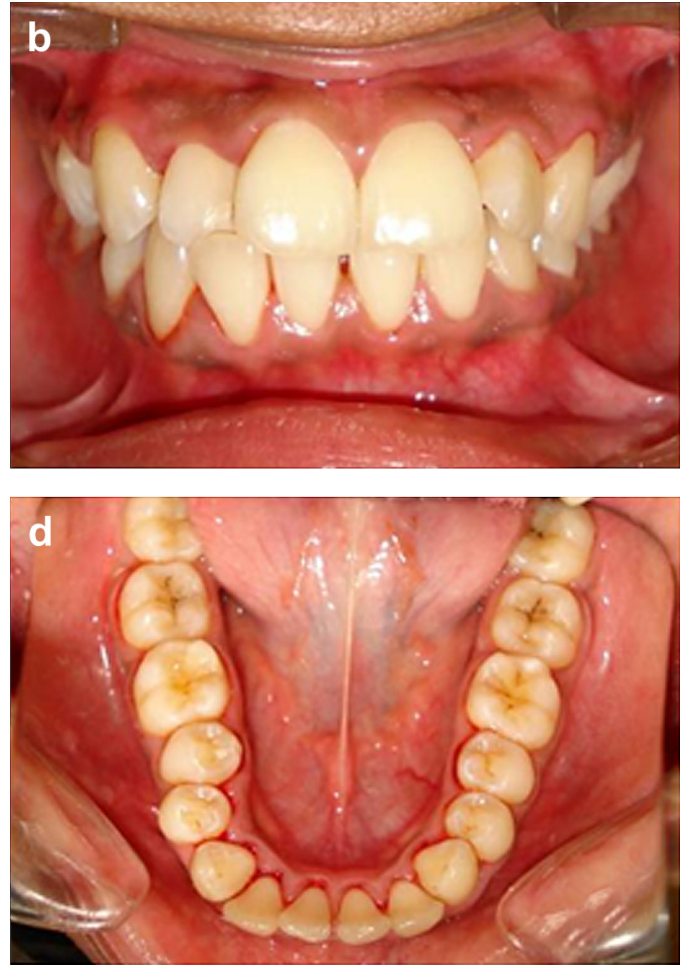

Fig. 1 - Pre-treatment photographs.

malocclusion. This ideal set up or template is then used as a physical guide to place the lingual brackets in an ideal configuration. The brackets are placed on the diagnostic set up using composite adhesive, which acts as a spacer between the metal mesh pad and the individual dental surfaces (Fig. 2). This process provides a finished result for the patient, almost identical to the finished ideal occlusion perfected in the laboratory, reducing significant clinical, chair-side archwire manipulation. Transfer trays are fabricated to facilitate indirect bonding of brackets.

\section{Appliance}

On completion of banding of molars, the entire set of self ligating brackets is bonded indirectly to the teeth anterior to the molars, with the exception of the premolars planned for therapeutic extraction, with trays using a light cured adhesive (Fig. 3a-c). A transpalatal bar may be used to augment maxillary posterior anchorage which is connected with a lingual sheath attachment on the molar band of the maxillary second molars. This transpalatal bar is usually placed posteriorly and shaped flat to form to the roof of the mouth to prevent discomfort to the patient.

The bite plane on the maxillary brackets maintains vertical control over the maxillary anterior teeth to reduce the previous gummy smile. To achieve final detailing of root angulations and the establishment of ideal anterior/posterior torque, $0.075^{\prime \prime} \times 0.025^{\prime \prime}$ TMA wires may be placed in both arches for 4-6 months. This was accomplished with tight bend backs behind the maxillary second molars. In the laboratory, the malocclusion was arranged to be that of the ideal occlusion. The brackets were placed on the ideal set up of the occlusion and later transferred to the patient's teeth. This process provides a finished result for the patient, which is very close to the finished ideal occlusion.

\section{Treatment results}

The dentition in both arches is brought within the limits of the facial oral muscles to establish good muscle balance and to improve pleasing esthetics. The teeth in habitual occlusion should have a Class I canine and molar relationship. Habitual occlusion should coincide with the centric relation occlusion. Both the maxillary and mandibular dental midlines should match with each other and the midpalatal suture. There must be good posterior intercuspation, with $2 \mathrm{~mm}$ overjet and overbite. The extraction sites in both arches must be closed (Fig. 4). Post-treatment evaluation should reveal no prematurities or mandibular shift. Bilateral canine and incisor guidance are desirable. The temporomandibular joints in occlusion must remain healthy.

\section{Retention}

The two areas of major concern for relapse were (1) the maintenance of lower incisor alignment and (2) closure of extraction sites. Patients who have chosen to have lingual braces are often esthetically demanding and do not like visible retainers. The maxillary and mandibular arches are retained with clear overlay retainers made of $0.4 / 0.5$ thick thermoplastic material, which are delivered on the same day of 

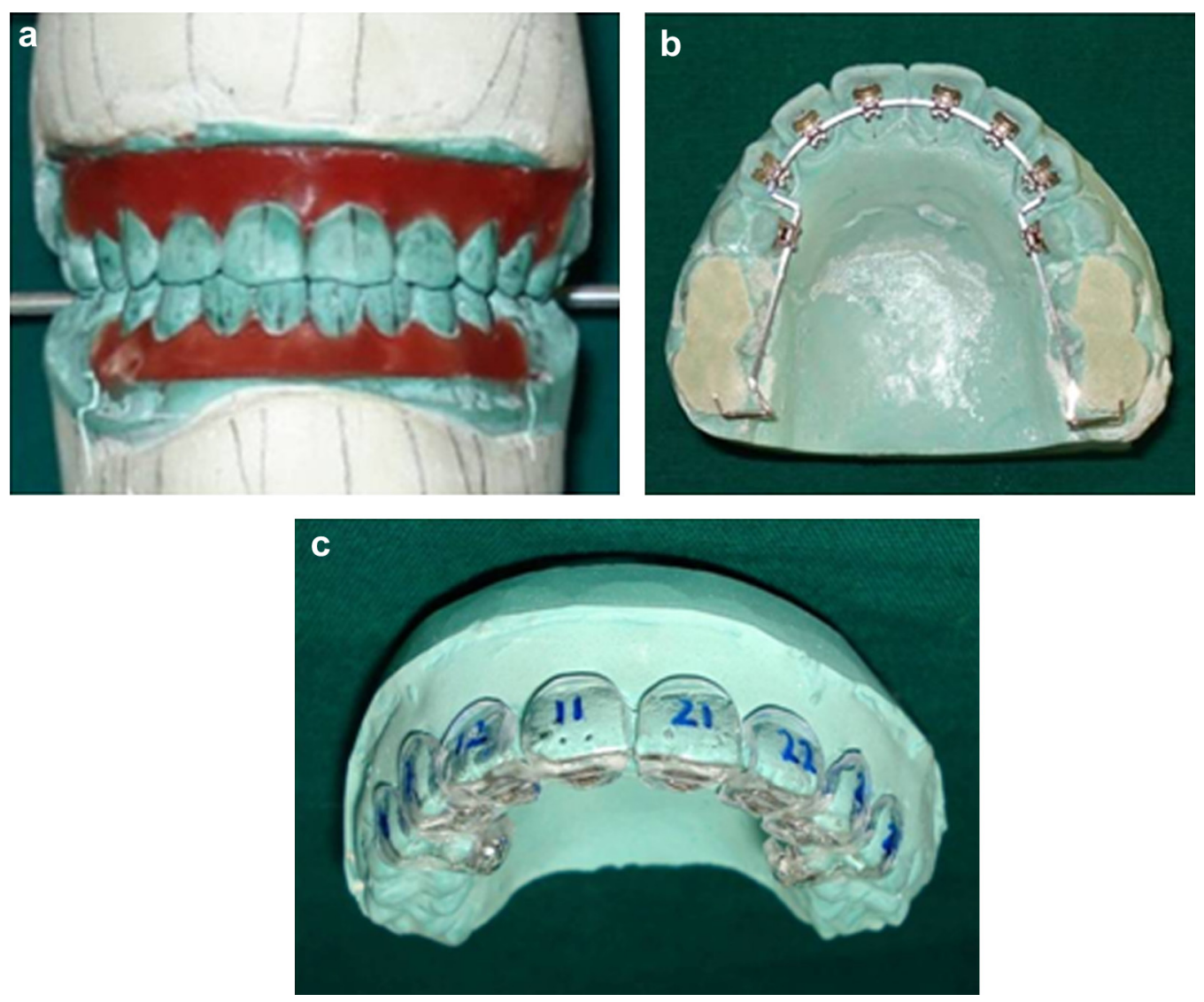

Fig. 2 - Complete Kesling set up with lingual brackets \& individual special trays.
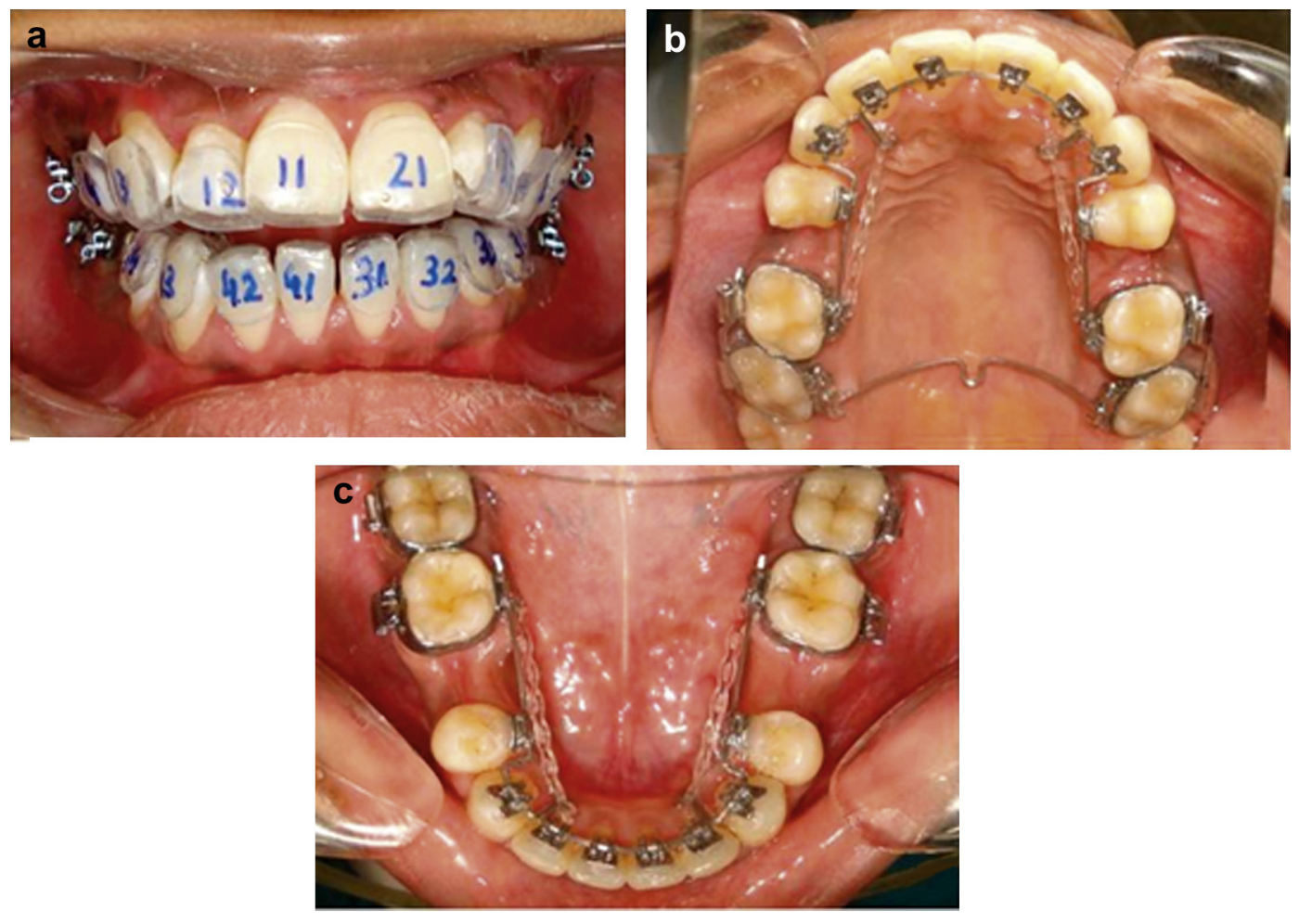

Fig. 3 - (a) Indirect bonding. (b) Palatal arch in situ. (c) Mandibular appliance. 

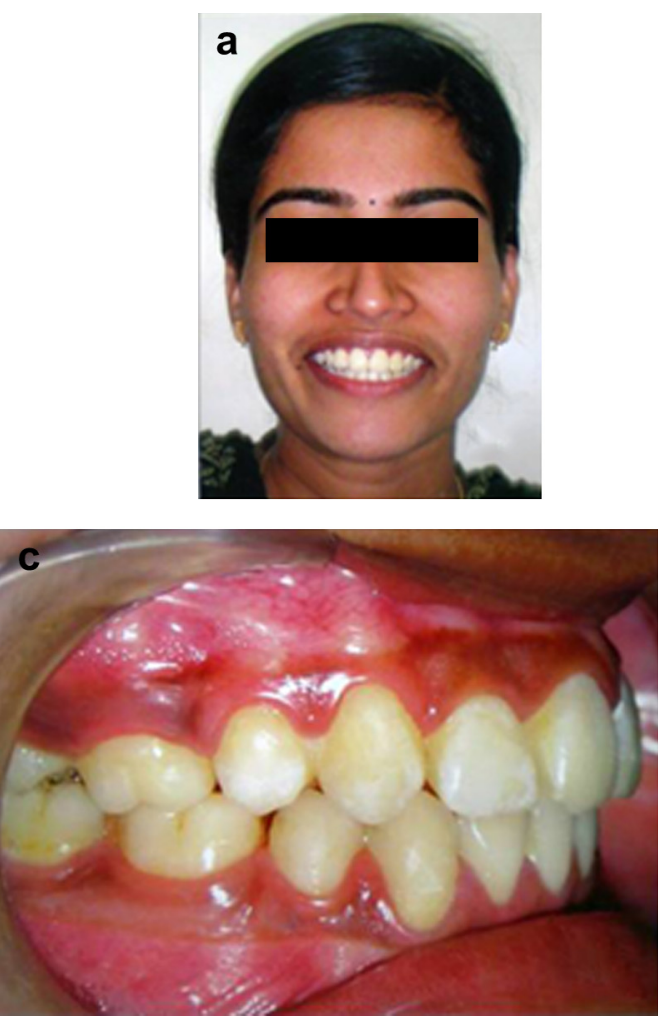
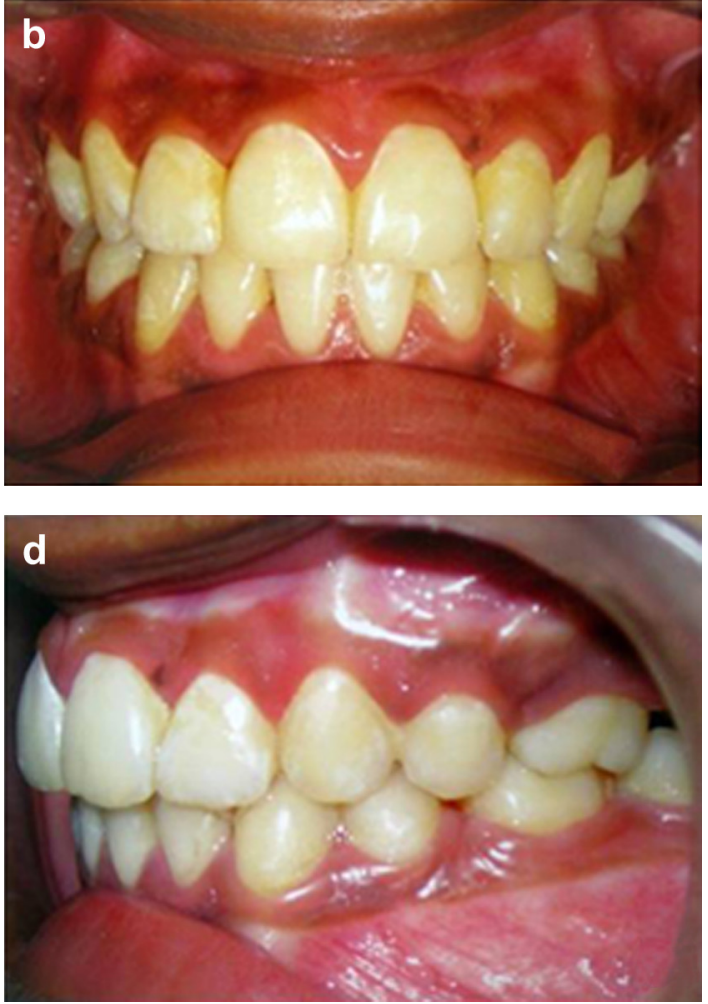

Fig. 4 - Post-treatment photographs.

appliance removal. The retainer is comfortable for the patient and allows for normal speaking with no wires crossing the occlusion. They are excellent for retaining alignment and provide exceptional esthetics.

\section{Other methods}

Intra-oral scanners are used to construct virtual models of teeth for fabricating customized precision lingual brackets and transfer jigs for indirect bonding with the aid of computer-aided design (CAD) \& computer-aided manufacturing (CAM) technology. However the cost of this technology is presently very prohibitive for providing affordable orthodontic therapy in India.

\section{Conclusion}

The lingual appliance has been shown to be as effective as its labial counterpart in correcting all types of malocclusion. ${ }^{3}$ The increased cost of this treatment coupled with the resistance on the part of many orthodontists to learn the new technique, seem to be the limiting factors of lingual orthodontics. ${ }^{4}$ Due to difficult access, limited visibility and space in the lingual region, self ligating lingual brackets offer the advantage of avoiding the tedious task of placing elastomeric or stainless steel ligature for securing archwires to the conventional ligating lingual brackets.
Most of the unique features of lingual orthodontics can be considered either as advantages or disadvantages, depending on the malocclusion and according to the specific goals of treatment. Teeth move under the force applied according to biomechanical principles and the biological environment.

\section{Conflicts of interest}

All authors have none to declare.

REFERENCES

1. Breece G, Nieberg L. Motivation for adult orthodontic treatment. J Clin Orthod. 1986;3:166-171.

2. Siures KP. Lingual and esthetic orthodontics. Br Dent J. 2011 Dec 23;211(12):614.

3. Grauer D, Proffit WR. Accuracy in tooth positioning with a fully customized lingual orthodontic appliance. Am J Orthod Dentofacial Orthop. 2011 Sep;140(3):433-443.

4. Fukawa $R$. Lingual orthodontics in the new era treatment according to criteria for occlusion and aesthetics. Int Orthod. 2009 Dec;7(4):370-402.

5. Hohoff A, Wiechmann D, Fillion D, Stamm T, Lippold C, Ehmer U. Evaluation of the parameters underlying the decision by adult patients to opt for lingual therapy: an international comparison. J Orofac Orthop. 2003 Mar;64(2):135-144. 